\title{
Karpal Tünel Sendromu Cerrahi Zamanlamasında EMG'nin Rolü
}

\author{
The Role of EMG in Surgical Timing of Carpal Tunnel \\ Syndrome
}

Bilsev Ince ${ }^{1}$

Zikrullah Baycar ${ }^{1}$

Tahsin Sami Çolak ${ }^{2}$

Mehmet Emin Cem Yıldırım

Mehmet Dadacı ${ }^{1}$

Mehmet Uyar ${ }^{3}$

İsmail Hakkı Korucu²

${ }^{1}$ Necmettin Erbakan Üniversitesi, Plastik Rekonstrüktif ve Estetik Cerrahi Anabilim Dalı, Konya, Türkiye

Necmettin Erbakan Üniversitesi, Ortopedi ve Travmatoloji Anabilim Dalı, Konya, Türkiye

${ }^{3}$ Necmettin Erbakan Üniversitesi, Halk

Sağlığı Anabilim Dalı, Konya, Türkiye

Geliş Tarihi/Received: 15 Ocak 2019

Kabul Tarihi/Accepted: 23 Şubat 2019

Yazışma Adresi: Bilsev İnce, Necmettin Erbakan Üniversitesi, Meram Tıp Fakültesi, Plastik Rekonstrüktif ve Estetik Cerrahi Anabilim Dalı, Konya, Türkiye

e-mail: bilsevince@yahoo.com

ORCID

Bilsev İnce

https://orcid.org/0000-0002-1756-4131

\begin{abstract}
Öz
Amaç: Karpal tünel sendromu (KTS), median sinirin el bileğinde karpal tünelde kompresyonu ile oluşan en sık görülen periferik mononöropatisidir. Bu çalışmada EMG'de ileti hızında azalma ve latansta uzama gösteren KTS tanılı hastalarda EMG verilerinin cerrahinin başarısı üstündeki rolü araştırıldı. Böylece cerrahi endikasyonların objektif kriterlere bağlanması amaçlandı.

Hastalar ve Yöntem: 2013-2018 yılları arasında elde uyuşma şikayetiyle olan, EMG'de karpal tünel sendromu tanısı almıs ve cerrahi uygulanmış hastalar prospektif olarak değerlendirildi. Ameliyat sonras 12.ayda hastaların şikayetlerinin devam edip etmediği sorgulanarak median sinirdeki ileti hızının değişimi yapılan EMG kontrolleri ile saptandı. Bu verilerle ameliyat öncesi EMG bulguları karşılaştırıldı. Ameliyat sonrası uyuşukluğun devam etmesi cerrahi başarısızlık olarak kabul edildi.

Bulgular: Çalışmaya 266 hasta (62 Erkek, 204 Kadın) dahil edildi. Şikayetleri devam eden hasta sayısı 23 'tü. Şikayeti devam eden hastaların median motor latans ortalaması 6,63 msn (min 3,04-max 11,04), ileti hızı ise ortalama $42,91 \mathrm{~m} / \mathrm{sn}(\min 36,88-\max 51,8$ )'di. Şikayetleri devam eden hastaların 16'sında median sinir duyu potansiyeli, 2'sinde hem duyu hem motor potansiyeli elde edilemedi. Şikayeti olmayan hastaların ise 70'inde median sinir duyu iletimi elde edilemezken, tamamında motor potansiyel elde edildi. Median motor sinir latans 4,63 msn'nin üstünde olduğunda KTS cerrahi tedavisinde hastanın uyuşukluk şikayetinin devam ettiği istatistiksel olarak tespit edildi.

Sonuç: Motor sinir Latans 4,63 msn'nin üstünde olduğunda KTS cerrahi tedavisinde başarı sansı azalmaktadır. KTS cerrahi tedavisi bu sınırlara gelmeden yapılması geri dönüşümsüz değişikliklerin
\end{abstract} ortaya çıkmasını engelleyebilir.

Anahtar Kelimeler: Elektromiyografi, Karpal tünel sendromu, Median nöropati

\begin{abstract}
Aim: Carpal tunnel syndrome (CTS) is the most common peripheral mononeuropathy caused by compression of the median nerve at the wrist inside the carpal tunnel. The aim of this study was to investigate the relationship between EMG data and surgical success in patients with CTS who showed a decrease in conduction velocity and prolonged latency in EMG. Thus, it was aimed to link the surgical indications to objective criteria.

Patients and Methods: The patients who were diagnosed with carpal tunnel syndrome and underwen surgery between 2013-2018 were evaluated prospectively. In the postoperative 12th month, changes in the median nerve velocity were observed by the EMG controls. Post-operative drowsiness was accepted as a surgical failure.

Results: 266 patients (62 males, 204 females) were included in the study. Complaints of 23 patients were continuing. The median motor latency average of the patients who complained was $6,63 \mathrm{msec}$ ( $\mathrm{min}$ 3,04- $\max 11,04$ ), and the message speed was 42,91 $\mathrm{m} / \mathrm{s}(\min 36,88$ - $\max 51,8)$. Median nerve sensory potential was not found in 16 patients, sensory and motor potential was not achieved in 2 patients. Median nerve sensory conduction could not be obtained in 70 of the patients without any complaint, but motor potential was obtained in all of patients. When median motor nerve latency was higher than 4.63 $\mathrm{ms}$, it was found that the patient complaint of drowsiness continued after surgical treatment of CTS Conclusion: When the motor nerve latans is above $4.63 \mathrm{msn}$, the chance of success in the surgical treatment of CTS decreases. Performing CTS surgical treatment before these limits may prevent the occurrence of irreversible changes.
\end{abstract}

Key words: Carpal Tunnel Syndrome, Electromyography, Median Neuropathy

\section{Gíriş}

En sık görülen periferik mononöropati olan karpal tünel sendromu (KTS), el bileğinde, karpal tünelde içinde oluşan basıya bağlı olarak median sinirin ilerleyici fonksiyon bozukluğu ile ortaya çıkan bir durumdur. Gece uyuşmasıyla başlayan tablo zamanla ilerleyici bir hal alarak uyuşukluğun kalıcı hale gelmesine yol açar (1). Geçmişteki çalışmalarda sıklığı \%1 olarak belirtilmesine rağmen (2) son yıllarda elektofizyolojik tanı imkanlarının artmasıyla beraber tanı oranı genel popülasyonda \%3.72 olarak belirtilmiştir (3). KTS'de tanı klinik semptomlar, fizik muayene bulguları ile konulabilse de kesin tanı elektro fizyolojik inceleme sonucu tespit edilir.
Atıf Yapmak İçin: Ince B, Baycar Z, Çolak TS, Yıldırım MEC, Dadacı M, Uya M, Korucu IH. Karpal Tünel Sendromu Cerrahi Zamanlamasında EMG'nin Rolü. Selcuk Med J 2019;35(2): 90-93
Açıklama: Yazarların hiçbiri, bu makalede bahsedilen herhangi bir ürün, aygıt veya ilaç ile ilgili maddi çıkar ilişkisine sahip değildir. Araștırma, herhangi bir dıs organizasyon tarafından desteklenmedi Yazarlar calışanı birincil verilerine tam erisim izni vermek ve derginin talep ettiği takdirde verileri incelemesine izin vermeyi kabul etmektedirler. 
Elektrofizyolojik testler, sadece kesin tanıyı koymakta değil, median sinirin tuzaklanma seviyesini belirleme ve bası ciddiyetini ortaya koymada da yararlıdır (4).

KTS tedavisinde erken evrelerde, konservatif tedavi tercih edilirken, dirençli olgularda veya orta ileri evrelerde cerrahi tedavi önerilmektedir (5-8). Konservatif tedavide antiinflamatuar tedavi, el ateli hareketsizlik ve fizik tedavi önerilmiştir (9). Ancak literatürde konservatif tedavi süresi ve içeriğiyle ilgili görüş birliği yoktur. Hafif ve orta KTS olgularının cerrahi girişimden daha fazla yarar sağladığı ileri sürülmüştür (10). KTS tanısı konulması için EMG'de sinir ileti hızının uzaması bir kriter olarak öne sürülmesine karşın ileti hızındaki uzamanın hastalığın şiddetine oranı tanımlanmamıştır. Bu çalışmada EMG'de ileti hızında azalma ve latansta uzama gösteren KTS tanılı hastalarda EMG verilerinin cerrahinin başarısı üstündeki rolü araştırıldı. Böylece cerrahi endikasyonların objektif kriterlere bağlanması amaçlandı.

\section{HASTALAR VE YÖNTEM}

2013-2018 yılları arasında hastanemize elde uyuşma şikayetiyle başvurup, EMG'de karpal tünel sendromu tanısı almış ve cerrahi uygulanmış hastalar prospektif olarak çalışmaya alındı. 6 hafta splintlemeye rağmen şikayetleri gerilemeyen veya splintleme sonrası şikayetlerinde tekrarlama olan ve üç hafta antinflamatuar tedavi sonrası şikayetleri devam etmesi cerrahi endikasyon olarak kabul edildi. Sigara ve/veya ilaç kullanan, gebeler, sistemik polinöropatiyle seyreden ek hastalığı olan hastalar ile kontrollere gelmeyen ve/veya kontrol EMG'si bulunmayan hastalar çalışma dışı bırakıldı. Çalışmaya Necmettin Erbakan Üniversitesi, Meram Tıp Fakültesi, Klinik Araştırmalar Etik Kurulu 2013/116 no'lu karar izniyle başlandı. Hastaların tamamında yazılı ve sözlü izin alındı.

Tüm hastalar lokal anestezi altında operasyona alındı. Cilt, cilt altı kesiyle karpal tünele ulaşılarak median sinir serbestlendi. Cilt, cilt altı primer kapatılıp günlük pansuman yapıldı. Hastalar 1., 7., 15. ve 30. Günlerde yara iyileşmesi açısından kontrol edildi. Ameliyat sonrası 12.ayda hastaların şikayetlerinin devam edip etmediği sorgulanarak median sinirdeki ileti hızının değişimi yapılan EMG kontrolleri ile saptandı. Bu verilerle ameliyat öncesi EMG bulguları karşılaştırıldı. Ameliyat sonrası uyuşukluğun devam etmesi cerrahi başarısızlık olarak kabul edildi. Hastaların ameliyat öncesi KTS nedeniyle iş güç kayıp gün sayısı ve cerrahi sonrası işe dönme zamanı sorgulandı.

\section{İstatistiksel Analiz}

İstatistiksel Analiz SPSS 24.0 versiyonu ile yapıldı. Median sinir latans hızının cerrahi müdahaleden fayda görecek hastaları öngörmede tanısal karar verdirici özellikleri Receiver Operating Characteristic (ROC) eğrisi analiz ile incelendi. Anlamlı sınır değerlerin varlığında bu sınırların sensitivite ve spesifisite değerleri hesaplandı. Eğri altında kalan alanın değerlendirilmesinde $\alpha<0.05$ olan durumlar testin tanısal değerinin istatistiksel olarak anlamlı olduğu şeklinde yorumlandı.

\section{BULGULAR}

Çalışmaya 266 (62 Erkek, 204 Kadın) dahil edildi. Hastaların yaş ortalaması 57.5 (29-81), ortalama takip süresi 17 aydı. Şikayetleri devam eden hasta sayısı 23, geçmiş olan hasta sayısı ise 243 idi. Şikayeti devam eden hastaların median motor latans ortalaması 6,63 msn ( $\min 3,04$ - $\max 11,04$ ), ileti hızı ise ortalama $42,91 \mathrm{~m} / \mathrm{sn}(\min 36,88-\max 51,8)$ idi. Hiçbir şikayeti olmayan hastaların median motor latans ortalaması 4,32 msn (min 2.9 - max 5.6), median motor hızı ortalamasının 53,1 m/sn (min 45 - max 58.7 $\mathrm{m} / \mathrm{sn}$ ) olduğu görüldü. Hastaların 86 'sında ameliyat öncesi yapılmış olan elektrofizyolojik incelemelerinde median sinir duyu iletimi elde edilemedi. Şikayetleri devam eden hastaların 16'sında median sinir duyu potansiyeli, 2'sinde hem duyu hem motor potansiyeli elde edilemedi. Şikayeti olmayan hastaların ise hastaların 70'inde median sinir duyu iletimi elde edilemezken, tamamında motor potansiyel elde edildi. Hastaların KTS nedeniyle konservatif tedavi aldığı dönemdeki iş güç kaybı ortalama 17 gün (10-23) iken, cerrahi sonrası ortalama işe başlama zamanı 12 gün (8-16) idi.

Median motor sinir latans 4,63 msn'nin üstünde olduğunda KTS cerrahi tedavisinde hastanın uyuşukluk şikayetinin devam ettiği istatistiksel olarak tespit edildi ( $p<0.001$, ilk tablo eğri altında kalan alan 0.828) (Şekil 1). Ancak median sinir motor ve duyu dalı ileti hızıyla hasta şikayetleri arasında istatistiksel anlamlı ilişki bulunamadı. Hastaların konservatif tedavi sırasında beyan ettikleri iş güç kaybı ise cerrahi sonrası ile karşılaştırıldığında istatistiksel olarak daha fazlaydı $(p<0.05)$.

\section{TARTIŞMA}

KTS median sinir sıkışmasının bilinen en sık şeklidir ve tüm tuzak nöropatilerinin \%90'ını oluşturur $(16,17)$. KTS sinirin karpal tünelde basıya uğraması 


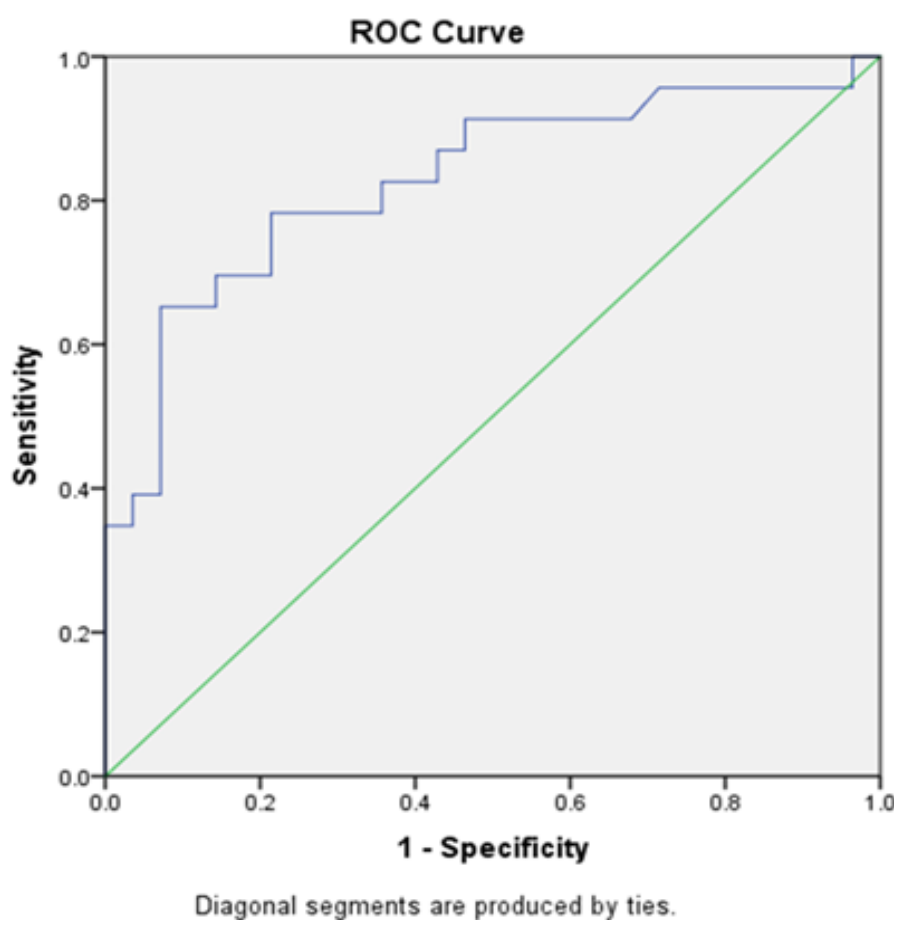

Şekil 1. Receiver Operating Characteristic (ROC) eğrisi analizinde anlamlı sınır değerlerin varlığında bu sınırların sensitivite ve spesifisite değerleri

sonucu gelişir. Bu bası sonrası mekanik olarak median sinir hasar görür ayrıca zamanla iskemi nedeniyle de median sinir geri dönüşümsüz zarar görebilir. Deneysel çalışmalar basınç miktarı ve süresi arttıkça sinir fonksiyonel bozulmasının da artacağını göstermiştir (18). Median sinir tekrar-layıcı mekanik strese maruz kaldığında demiyelinizasyon gelişir (19) zamanla kan sinir bariyerinde bozulma ve aksonal hasar meydana gelir (20). Bası uzun sürdüğünde hasar geri dönüşümsüz olabilir. Çalışmamızda da ameliyat öncesi çekilen EMG'de motor hızında kayıp arttığında hastaların cerrahi tedaviden yarar göremediği/kısmen yarar gördüğü görüldü.

KTS tanısında anamnez, fizik muayene, radyolojik görüntülemeler ve elektrofizyolojik testler kullanılmaktadır. Median sinirin uzamış motor ve duyu distal latansı, azalmış motor ve duyu hızları tanı kriteri olarak tanımlanmıştır (21). KTS tedavisi, konservatif ve cerrahi tedavi olmak üzere iki kategoriye ayrılır. Hafif ve orta KTS olgularında konservatif tedavi yöntemleri önerilmesine rağmen (22) bazı çalışmalarda konservatif tedavilerin kısa süreli önemli faydası olsa da uzun süreli etkinliklerinin tartışmalı olduğu bildirilmiştir (23). Randomize kontrollü bir çalışmada en az 6 hafta splint uygulamak ile cerrahi karşılaştırımış ve cerrahinin daha etkili olduğu gösterilmiştir (25). Başka bir çalışmada ise splint tedavisi ile cerrahi tedavi benzer maliyete sahip olsa da ( ortalama 2100 EURO) etkinlikleri sıraya \%72'ye karşı \%92 bulunmuş ve cerrahinin daha maliyet etkin olduğu bulunmuştur (26). Ayrıca splint tedavisi alan hastaların \% 39'u 12 ay sonra cerrahi tedaviye intiyaç duymuştur (26). Tek doz steroid tedavisi ile cerrahi tedaviyi karşılaştıran randomize bir çalışmada cerrahi tedavinin semptomları anlamlı olarak daha fazla azalttığı gösterilmiştir. Aynı çalışmada cerrahi grubun median sinir distal motor latansı ortalaması 5.4 iken cerrahi sonrası 4.2'ye düştüğü ve streoid tedavisine göre daha iyi iyileşme sağladığı gösterilmiştir (27).

Cerrahi tedavide karpal ligaman kesilerek median sinir üzerindeki bası ortadan kaldırılır. Cerrahi sonrası uzun dönem sonuçlarına bakıldığında \%70-90 arası başarılı sonuçlar elde edilmiştir (24). Literatürde cerrahi tedavi için açık, mini açık ve endoskopik yöntemler tarif edilmiş olup bu yöntemler karşılaştırılmıştır $(11,12)$. Transvers karpal ligamentin gevşetilmesi ve karpal tünelin dekompresyonu ile birçok hasta klinik olarak iyileşse de bazı hastaların yakınması devam etmektedir (12). Çok merkezli bir çalışmada cerrahi girişim sonrası hastaların \%10-15'inde şikayetlerin hiç geçmediği veya erken dönemde tekrarladığı görülmüştür (13). Bazı çalışmarda ise bu oran \%1.625 arasında bulunmuştur $(14,15)$. Ancak bu hastaların EMG bulguları ve KTS'nin seviyesi bildirilmemiştir. Bu nedenle başarısızlık olan hastaların ne kadarının median sinirdeki basının oluşturduğu kalıcı hasara, ne kadarının yetersiz cerrahi gevşetmeye bağlı olduğu belirlenememiştir.

Hafif ve orta evre KTS hastalarında cerrahi tedavi sonuçlarının ağır KTS hastalarına göre daha iyi olduğu bildirilmiştir. Bizim çalışmamızda da EMG median sinir motor distal latansı üzerinde olan ve motor ileti hızı altında olan hastalarda cerrahi sonrası başarı oranı anlamlı olarak azalmaktadır. Literatürde cerrahi girişim sonrası şikayetlerin devam etmesi ile ilgili olarak yetersiz gevşetme, proksimalde başka bir nöropatinin varlığı gibi sebepler gösterilmiştir. Ancak çalışmamızda başka bir nöropatisi olmayan KTS tanısı konulmuş hastalarda cerrahi öncesi elektrofizyolojik değerleri ile cerrahi başarısı arasında ilişki olduğu istatistiksel olarak tespit edildi. Ameliyat için fizik tedavi, splintleme veya medikal tedavi için geçirilen süre belirli sınırı aştığında cerrahi başarı şansı geri dönüşümsüz olarak azalmaktaydı. Bu nedenle KTS tanısında en objektif tanı yöntemi 
olan EMG sonuçlarına göre konservatif tedavi ile zaman harcanmadan lokal anestezi altında minimal kesiyle yapılabilinecek cerrahi gevşetmenin kalıcı hasarı engelleyebileceğini düşünüyoruz. KTS cerrahi tedavisi ülkemizde 90 dolar olup, hasta bir hafta sonra işe başlayabilmektedir. Cerrahi için tarafımıza başvuran hastalarda ise bu süre ortalama 13 gün idi. KTS tanısının koyulması ile cerrahi yapılma arasındaki süre arttıkça hastada geri dönüşümsüz hasar riskinin artmaktadır ayrıca hastaların şikayetleri nedeniyle yaşadıkları iş güç kaybı süresi cerrahi sonrasından istatistiksel olarak daha fazladır. Bu süreler de dikkate alındığında KTS'da erken cerrahi girişimin faydası ortaya çıkmaktadır.

$\mathrm{Bu}$ çalışma KTS cerrahi zamanlamasının belirlenmesinde EMG verilerinin sınırlarının objektif olarak ortaya konduğu literatürdeki ilk çalışmadır. Motor sinir latans 4,63 msn'nin üstünde olduğunda KTS cerrahi tedavisinde başarı şansı azalmaktadır. KTS cerrahi tedavisi bu sınırlara gelmeden yapılması geri dönüşümsüz değişikliklerin ortaya çıkmasını engelleyebilir.

Çıkar Çatışması: Çalışmada herhangi bir çıkar çatışması yoktur.

Finansal Çıkar Çatışması: Çalışmada herhangi bir finansal çıkar çatışması yoktur.

Yazışma Adresi: Bilsev Ince, Necmettin Erbakan Üniversitesi, Meram Tıp Fakültesi, Plastik Rekonstrüktif ve Estetik Cerrahi Anabilim Dalı, Konya, Türkiye

e-mail: bilsevince@yahoo.com

\section{KAYNAKLAR}

1. Sucher BM, Schreiber AL. Carpal tunnel syndrome diagnosis. Phys Med Rehabil Clin N Am 2014;25:229-47.

2. Pfeffer GB, Gelberman RH, Boyes JH, et al. The history of carpal tunnel syndrome. J Hand Surg [Br] 1988;13:28-34.

3. Papanicolaou GD, McCabe SJ, Firrell J. The prevalence and characteristics of nerve compression symptoms in the general population. J Hand Surg [Am] 2001;26:460-6.

4. Sen D, Chhaya S, Morris VH. Carpal tunnel syndrome. Hospital Medicine 2002;63;7:392-5.

5. Mishra S, Prabhakar S, Lal V, et al. Efficacy of splinting and oral steroids in the treatment of carpal tunnel syndrome: $A$ prospective randomized clinical and electrophysiological study. Neurol India 2006;54(3):286-90.

6. Diagnoses and Treatment of Work Related Carpal Tunnel Syndrome (OCTS). The Washington State Medical Associati on (WSMA) Medical Treatment Guidelines Subcommittee and the Department of Labor and Industries Office of the Medical Director. The Medical Treatment Guidelines 2004. p. $32-40$

7. Moraska A, Chandler C, Edmiston SA, et al. Comparison of a targeted and general massage protocol on strength, function, and symptoms associated with carpal tunnel syndrome: A randomized pilot study. $\mathrm{J}$ Al tern Complement Med 2008;14(3):259-67.

8. Baysal O,Altay Z, Ozcan C, et al. Comparison of three conservative treatment protocols in carpal tunnel syndrome. Int J Clin Pract 2006;60(7):820-8.

9. American Academy of Orthopaedic Surgery (AAOS). Clinical practice guideline on the treatment of carpal tunnel syndrome. 2016.

10. Alemdar M. Turkiye Klinikleri J Neur 2009;4(1):19-32.

11. Cokluk $C$, Senel $A$, Iyigun $O$, et al. Open median nerve release using double mini skin incision in patients with carpal tunnel syndrome: Technique and clinical results. Neurol Med Chir (Tokyo) 2003;43:465-8.

12. Tetik B, Erol B. Karpal tünel sendromunun cerrahi tedavisinde uygulanan alternatif metodların karşılaştırılması. J Arthroplasty\& Arthrosopic Surg 2002;13(1):5-9.

13. Brown RA, Gelberman RH, Seiler JG, et al. Carpal tunnel release. A prospective, randomized assessment of open and endoscopic methods. J Bone Joint Surg [Am] 1993;7:126575.

14. Langloh ND, Linscheid RL. Recurrent and unrelieved carpal tunnel syndrome. Clin Orthop 1972;83:41-7.

15. Kulick MI, Gordillo G, Javidi T, et al. Longterm analysis of patients having surgical treatment for carpal tunnel syndrome. J Hand Surg [Am] 1986;11:59-66.

16. American Academy of Orthopaedic Surgeons Work Group Panel. Clinical guidelines on diagnosis of carpal tunnel syndrome, 2007.

17. Aroori S, Spence RA. Carpal tunnel syndrome. Ulster Medical J 2008;77(1) 6-17.

18. Mackinnon SE. Pathophysiology of nerve compression. Hand Clin 2002; 18(2): 231-41.

19. Alfonso C, Jann S, Massa R, et al. Diagnosis, treatment and followup of the carpal tunnel syndrome: A review. Neurolog Sci 2010; 31(3): 243-52.

20. Burns TM. Mechanisms of acute and chronic compression neuropathy. In: Dyck PJ, Thomas PK, Eds. Peripheral neuropathy. 4th ed. Amsterdam: Elsevier 2005; pp. 1391402.

21. Swash M, Schwarts M. Nerve entrapment and compression syndromes and other mono neuropathies. Berlin: Springer 1997.

22. Prime MS, Palmer J, Khan WS, et al. Is there light at the end of the Tunnel? Controversies in the diagnosis and management of carpal tunnel syndrome. Hand 2010;5(4):35460.

23. O'Connor D, Marshall S, Massy Westropp N. Non surgical treatment (other than steroid injection) for carpal tunnel syndrome. Cochrane Database Syst Rev 2003;(1):CD003219.

24. Turner A, Kimble F, Gulyas K, et al. Can the outcome of open carpal tunnel release be predicted?: A review of the literature. ANZ J Surg 2010;80:50-4.

25. Gerritsen AAM, de Vet HCW, Scholten RJPM, et al. Splinting vs surgery in the treatment of carpal tunnel syndrome: $A$ randomized controlled trial JAMA. 2002;288(10):1245-51.

26. Korthals-de Bos IB, Gerritsen AA, van Tulder MW, et al. Surgery is more costeffective than splinting for carpal tunnel syndrome in the Netherlands: results of an economic evaluation alongside a randomized controlled trial. BMC Musculoskeletal Disorders 2006; 16;7:86.

27. Hui $\mathrm{AC}$, Wong $\mathrm{S}$, Leung $\mathrm{CH}$, et al. A randomized controlled trial of surgery vs steroid injection for carpal tunnel syndrome. Neurology. 2005;64(12):2074-8. 